\title{
Metabolic responses of Carlina acaulis L. to chronic and acute cadmium stress: insights into chelation mechanisms, non-enzymatic antioxidants, and specialized metabolism
}

Sławomir Dresler ( $\nabla$ slawomir.dresler@poczta.umcs.lublin.pl )

Uniwersytet Marii Curie-Sklodowskiej https://orcid.org/0000-0003-4413-3748

Maciej Strzemski

Uniwersytet Medyczny w Lublinie

Jozef Kováčik

Trnavska Univerzita v Trnave

Jan Sawicki

Uniwersytet Medyczny w Lublinie

Michał Staniak

Uniwersytet Medyczny w Lublinie

Magdalena Wójciak

Uniwersytet Medyczny w Lublinie

Ireneusz Sowa

Uniwersytet Medyczny w Lublinie

\section{Barbara Hawrylak-Nowak}

Uniwersytet Przyrodniczy w Lublinie

Research article

Keywords: antioxidants, trace metals, phenolic compounds, chelation, terpenoids

Posted Date: March 29th, 2020

DOI: https://doi.org/10.21203/rs.2.20457/v2

License: (c) (i) This work is licensed under a Creative Commons Attribution 4.0 International License.

Read Full License 
The authors have withdrawn this preprint from Research Square 